\title{
Non-urothelial Bladder Cancers: Single Center Experience
}

\author{
(D) Abdullah Sakin1, (D) Şaban Seçmeler², (D) Serdar Arıcı², (D) Orçun Can², (D) Çağlayan Geredeli2, (D) Nurgül Yaşar², (D) Cumhur Demir² \\ 1 Van Yüzüncü Yıl University Faculty of Medicine, Department of Medical Oncology, Van, Turkey \\ 2istanbul Okmeydanı Training and Research Hospital, Clinic of Medical Oncology, Istanbul, Turkey
}

\section{Abstract}

Objective: Bladder cancers are classified into two groups as urothelial and non-urothelial cancer (NUBC). In this study, we investigated the clinicopathological features and treatment responses in patients with NUBC who were diagnosed and treated in our center.

Methods: The patients who were diagnosed and treated in our hospital between 2006 and 2017 were included in the study retrospectively. Patients with urothelial or mixed bladder cancers were excluded from the study.

Results: A total of 16 patients with NUBC were included in the study. Six patients had squamous cell carcinoma, six patients had small cell carcinoma and four patients had adenocarcinoma. Thirteen patients (81\%) were male and three were female. Although there was no statistically significant difference between the groups in terms of localization of metastases $(p=0.663)$, the most common site of metastasis was pelvic structures in squamous cell carcinoma group and multiple distant organ metastasis were observed in small cell carcinoma group. The median survival in squamous cell carcinoma, adenocarcinoma, and small cell carcinoma was $11 \pm 5.3,12 \pm 10$, and $2 \pm 1.8$ months, respectively (logrank $p=0.329$ ).

Conclusion: There are no large prospective randomized clinical studies to guide treatment in NUBC. In the light of these results, we recommend that these patients should participate in prospective clinical studies.

Keywords: Squamous cell cancer, small cell cancer, bladder cancer, chemotherapy

\section{INTRODUCTION}

Bladder cancers are the most frequently detected cancers of the genitourinary system in men and women. Bladder cancers are divided into two groups as urothelial and non-urothelial cancer (NUBC). Although the pathogenesis of NUBC has not been clarified, the main reason is thought to be infection and inflammation leading to metaplasia of epithelial cells (1). NBUCS are rare and consist of a histologically heterogeneous group, so there is no clear treatment approach. Appropriate treatment approaches were investigated with retrospective studies and small-scale prospective studies. Cystectomy and lymph node dissection are recommended in patients with early-stage adenocarcinoma (AC), squamous cell carcinoma (SqCC) and NUBC associated with schistosomiasis (2). Best supportive care, chemotherapy (CT), and radiotherapy (RT) are the treatment options for metastatic and inoperable NUBCs. The studies about targeted therapy based on tumor-specific mutations are ongoing in this group (3). We investigated the factors in the pathogenesis of disease, clinicopathological features of patients and responses to treatments in patients with NUBC who were diagnosed and treated in our center.

\section{METHODS}

The patients who were diagnosed and treated in our hospital between 2006 and 2017 were included in this study retrospectively. Patients with urothelial or mixed bladder cancer and non-bladder cancer were excluded from the study. A total of 16 patients were included in the study. Ethics committee approval was granted for the study.

\section{Statistical Analysis}

Statistical Package for the Social Sciences (SPSS) 15.0 for Windows was used for statistical analysis. Descriptive statistics were given as mean, standard deviation, minimum and maximum for numerical variables, and as number and percentages for 
categorical variables. The ratios were compared using chi-square test. Monte Carlo simulation was used when conditions were not met. Survival analysis was performed using Kaplan Meier analysis. The statistical significance alpha level was accepted as $\mathrm{p}<0.05$.

\section{RESULTS}

A total of 16 patients with NUBC, including six patients with $\mathrm{SqCC}$, six patients with small cell carcinoma (SCC), and four patients with AC, were included in the study. Thirteen patients (81\%) were male and three (19\%) were female. The mean age was $62.5 \pm 10.6$ years. There was no statistically significant difference between the groups regarding gender and age $(p=0.744$ and $p=0.743$, respectively). A statistically significant difference was detected between the Eastern Cooperative Oncology group (ECOG) performance scores $(p=0.011)$. The ECOG performance score of three SCC patients (66.3\%) was 3. The ECOG performance score of three SqCC patients (50\%) was 3. There was no statistically significant difference between the groups in terms of smoking $(p=0.141)$. Smoking was a predisposing factor in two patients (12.5\%) and chronic urinary infection was a predisposing factor in one patient (6.3\%). There was no significant difference between the groups in terms of grade and stages $(p=0.236, p=0.109)$. Ten patients (62.5\%) had stage 4 disease at diagnosis. Five SCC patients (83.3\%) had stage 4 disease at diagnosis. The most common site of metastasis was pelvic structures in all patients. Although there was no statistically significant difference between the groups in terms of metastasis site ( $p=0.663$ ), the most common site of metastasis was pelvic structures in the SqCC group, but patients with SCC had multiple distant organ metastasis. Four patients (25\%) underwent radical surgery and received postoperative adjuvant therapy. Local RT was performed in 8 patients (50\%). Five patients (31.2\%) received platin (cisplatin or carboplatin) plus gemcitabine CT, 3 patients (18.9\%) received platin (cisplatin or carboplatin) plus etoposide (PE), one patient (6.3\%) received platin (cisplatin or carboplatin) plus 5-fluorouracil, one patient (6.3\%) received etoposide alone and one patient (6.3\%) received gemcitabine alone. Thirteen patients (81.3\%) died during the follow-up (Table 1). The median overall survival (OS) was $10 \pm 4.6$ months for all patients and the median OS was $11 \pm 5.3,12 \pm 10$ and $2 \pm 1.8$ months for SqCC, AC, and SCC, respectively (Table 2). There was no statistically significant difference between the groups in terms of OS (logrank $p=0.329$ ) (Figure 1).

\section{DISCUSSION}

NUBCs are less than $5 \%$ of all bladder cancers. Approximately $90 \%$ of NUBCs are epithelial cancers that may include SqCC, AC, and SCC. Non-epithelial tumors include sarcoma, carcinoma, paraganglioma, melanoma, and lymphomas (1). Other histological forms of bladder cancer are frequently observed seen in NUBCs. More than one tumor type was detected in 44 patients (68\%) in a series of 64 patients and urothelial carcinoma (UC) component was detected in 38 patients (58\%) (4). Similarly, in another study, a total of 51 patients with SCC were included and it was reported that the rates of simultaneously detected UC, AC and SqCC were $70 \%, 8 \%$ and $10 \%$, respectively (5). We did not include any patients with mixed histology in our study. Six patients with pure SqCC, four patients with pure $A C$ and six patients with pure SCC were included in this study. Chronic urinary tract infections are associated with bladder cancer. Infection can contribute to the development of bladder cancer through multiple mechanisms. SqCCs account for 3-5\% of bladder cancers in North America and Europe. SqCC is most frequently associated with squamous metaplasia and is detected in 16-28\% of patients with leukoplakia (6). However, in tendemic regions for Schistosoma haematobium infection, SqCC accounts for $75 \%$ of bladder cancers. Other risk factors for SqCC are bladder stone, pelvic RT, history of intravesical Bacillus Calmette-Guerin treatment, long-term use of cyclophosphamide and smoking $(1,7)$. In our study, five SqCC patients had smoking history and one patient had chronic urinary tract infection. The predisposing factors in our study were smoking in two patients (12.5\%) and chronic urinary tract infection in one patient (6.3\%). The optimal treatment of non-metastatic SqCC is surgery. Preoperative RT is an acceptable option for local advanced disease. In a study in a total of 1422 male patients with SqCC, it was reported that the 2-year all-cause mortality rates were $11 \%$ in stage 1 disease and $72 \%$ in stage 4 disease. SqCC histology was associated with poorer outcomes compared with urothelial bladder cancer, and local recurrence was most frequently detected in SqCC (8). In Phase 3 BC2001 study, researchers observed that simultaneous administration of fluorouracil and mitomycin C with RT improved the local control and survival in patients with muscleinvasive bladder cancer. However, only $2.7 \%$ of the patients were diagnosed with $\mathrm{AC}$ or SqCC in this study (9). Limited data suggested that $\mathrm{SqCC}$ was relatively more resistant to $\mathrm{CT}$ regimens used in metastatic UC (10). In our study, 3 patients (50\%) with SqCC were stage 2 . Only one patient (16.7\%) underwent curative surgery and was administered adjuvant cisplatin + gemcitabine treatment. RT was given to 3 patients (50\%) at the time of diagnosis. Three patients had metastatic disease at the time of diagnosis, and all received palliative CT (platin+gemcitabine). Four patients died at a median follow-up of 12.8 months (range, 10.5-33.2). The median OS in SqCC was 11 months. AC is lower than $2 \%$ of all bladder cancers in North America and Europe. 


\begin{tabular}{|c|c|c|c|c|c|c|c|c|c|c|}
\hline & & \multirow[b]{2}{*}{$n$} & \multirow[b]{2}{*}{$\%$} & \multicolumn{2}{|c|}{$\mathrm{SqCC}$} & \multicolumn{2}{|c|}{$\mathrm{AC}$} & \multicolumn{2}{|c|}{ SCC } & \multirow[b]{2}{*}{$p$} \\
\hline & & & & $n$ & $\%$ & $\mathrm{n}$ & $\%$ & $n$ & $\%$ & \\
\hline \multirow[t]{2}{*}{ Gender } & Male & 13 & 81.3 & 4 & 66.7 & 4 & 100.0 & 5 & 83.3 & 0.744 \\
\hline & Female & 3 & 18.8 & 2 & 33.3 & 0 & 0.0 & 1 & 16.7 & \\
\hline Age (years) & $\begin{array}{l}\text { Mean } \pm \text { SD } \\
\text { (min-max) }\end{array}$ & \multicolumn{2}{|c|}{$\begin{array}{l}62.5 \pm 10.6 \\
(43-81)\end{array}$} & \multicolumn{2}{|c|}{$\begin{array}{l}61.0 \pm 11.2 \\
(52-80)\end{array}$} & \multicolumn{2}{|c|}{$\begin{array}{l}62 \pm 12.1 \\
(43-70)\end{array}$} & \multicolumn{2}{|c|}{$\begin{array}{l}64 \pm 10.7 \\
(49-81)\end{array}$} & 0.743 \\
\hline \multirow{5}{*}{ ECOG PS } & 0 & 2 & 12.5 & 0 & 0.0 & 0 & 0.0 & 2 & 33.3 & 0.011 \\
\hline & 1 & 4 & 25.0 & 2 & 33.3 & 2 & 50.0 & 0 & 0.0 & \\
\hline & 2 & 4 & 25.0 & 3 & 50.0 & 1 & 25.0 & 0 & 0.0 & \\
\hline & 3 & 4 & 25.0 & 0 & 0.0 & 0 & 0.0 & 4 & 66.7 & \\
\hline & 4 & 2 & 12.5 & 1 & 16.7 & 1 & 25.0 & 0 & 0.0 & \\
\hline \multirow{2}{*}{ Smoking } & Yes & 11 & 68.8 & 4 & 66.7 & 4 & 100.0 & 3 & 50.0 & 0.141 \\
\hline & No & 5 & 31.3 & 2 & 33.3 & 0 & 0.0 & 3 & 50.0 & \\
\hline \multirow{3}{*}{ Predisposing factors } & Chronic UTI & 1 & 6.3 & 1 & 16.7 & 0 & 0.0 & 0 & 0.0 & 0.518 \\
\hline & Smoking & 2 & 12.5 & 1 & 16.7 & 1 & 25.0 & 0 & 0.0 & \\
\hline & None & 13 & 81.3 & 4 & 66.7 & 3 & 75.0 & 6 & 100.0 & \\
\hline \multirow{2}{*}{ Grade } & 2 & 8 & 50.0 & 4 & 66.7 & 3 & 75.0 & 1 & 16.7 & 0.236 \\
\hline & 3 & 8 & 50.0 & 2 & 33.3 & 1 & 25.0 & 5 & 83.3 & \\
\hline \multirow{3}{*}{ TNM stage } & 2 & 4 & 25.0 & 3 & 50.0 & 0 & 0.0 & 1 & 16.7 & 0.109 \\
\hline & 3 & 2 & 12.5 & 0 & 0.0 & 2 & 50.0 & 0 & 0.0 & \\
\hline & 4 & 10 & 62.5 & 3 & 50.0 & 2 & 50.0 & 5 & 83.3 & \\
\hline \multirow{5}{*}{ Metastasis sites at diagnosis } & Lymph node & 1 & 10.0 & 0 & 0.0 & 0 & 0.0 & 1 & 20.0 & 0.663 \\
\hline & Pelvic structures & 5 & 50 & 2 & 66.7 & 1 & 50.0 & 1 & 40.0 & \\
\hline & Bone & 1 & 10.0 & 0 & 0.0 & 1 & 50.0 & 0 & 0.0 & \\
\hline & Lung & 1 & 10.0 & 1 & 33.3 & 0 & 0.0 & 0 & 0.0 & \\
\hline & Multiple & 2 & 20.0 & 0 & 0.0 & 0 & 0.0 & 2 & 40.0 & \\
\hline \multirow{2}{*}{ Radical surgery } & No & 12 & 75.0 & 5 & 83.3 & 2 & 50.0 & 5 & 83.3 & 0.470 \\
\hline & Yes & 4 & 25.0 & 1 & 16.7 & 2 & 50.0 & 1 & 16.7 & \\
\hline \multirow{3}{*}{ Chemotherapy } & Adjuvant at diagnosis & 4 & 25.0 & 1 & 16.7 & 2 & 50.0 & 1 & 16.7 & 0.959 \\
\hline & Palliative at diagnosis & 7 & 43.8 & 3 & 50 & 1 & 25.0 & 3 & 50.0 & \\
\hline & None & 5 & 31.2 & 2 & 33.3 & 1 & 25.0 & 2 & 33.3 & \\
\hline \multirow{3}{*}{ RT } & At diagnosis & 8 & 50.0 & 3 & 50.0 & 3 & 75.0 & 2 & 33.3 & 0.614 \\
\hline & Relapse/progress & 1 & 6.3 & 1 & 16.7 & 0 & 0.0 & 0 & 0.0 & \\
\hline & None & 7 & 43.8 & 2 & 33.3 & 1 & 25.0 & 4 & 66.7 & \\
\hline \multirow{5}{*}{ Chemotherapy } & Etoposide & 1 & 6.3 & 0 & 0.0 & 0 & 0.0 & 1 & 16.7 & 0.527 \\
\hline & Platin + flourourasil & 1 & 6.3 & 1 & 16.7 & 1 & 25.0 & 0 & 0.0 & \\
\hline & Platin + etoposide & 3 & 18.9 & 0 & 0.0 & 0 & 0.0 & 3 & 52.2 & \\
\hline & Platin + gemcitabine & 5 & 31.2 & 4 & 66.7 & 1 & 25.0 & 0 & 0.0 & \\
\hline & Gemcitabine & 1 & 6.3 & 0 & 0.0 & 1 & 25.0 & 0 & 0.0 & \\
\hline \multirow{2}{*}{ Final status } & Alive & 3 & 18.8 & 2 & 33.3 & 0 & 0.0 & 1 & 16.7 & 0.738 \\
\hline & Dead & 13 & 81.3 & 4 & 66.7 & 4 & 100.0 & 5 & 83.3 & \\
\hline
\end{tabular}


Glandular, colloidal, signet-ring and clear cell variants have been described. The signet-ring cell variant is associated with poor prognosis. Metastasis is more frequently detected in $A C$ than SqCC. Unlike UC, local recurrence is more common than distant metastasis (11). Surgery is the optimal treatment for AC. Partial cystectomy may be an alternative to radical cystoprostatectomy in patients with urachal AC. Partial cystectomy is not recommended in patients with non-urachal AC. There is no available randomized study focused on neoadjuvant or adjuvant $\mathrm{CT}$. The role of $\mathrm{CT}$ or RT is not clear in patients with local advanced or metastatic AC (12). In a study, it was found that postoperative RT was associated with longer relapse-free survival and smaller local recurrence rate, but with a higher rate of distant metastases (13). In another prospective study, male patients with advanced stage NUBC were included and 11 patients had AC histology. Patients received ifosfamide, paclitaxel and cisplatin. Response rate was 36\% and the median OS was 25 months (14). In another retrospective study of 14 patients with $\mathrm{AC}$, the response rate was $36 \%$ for regimen containing first-line

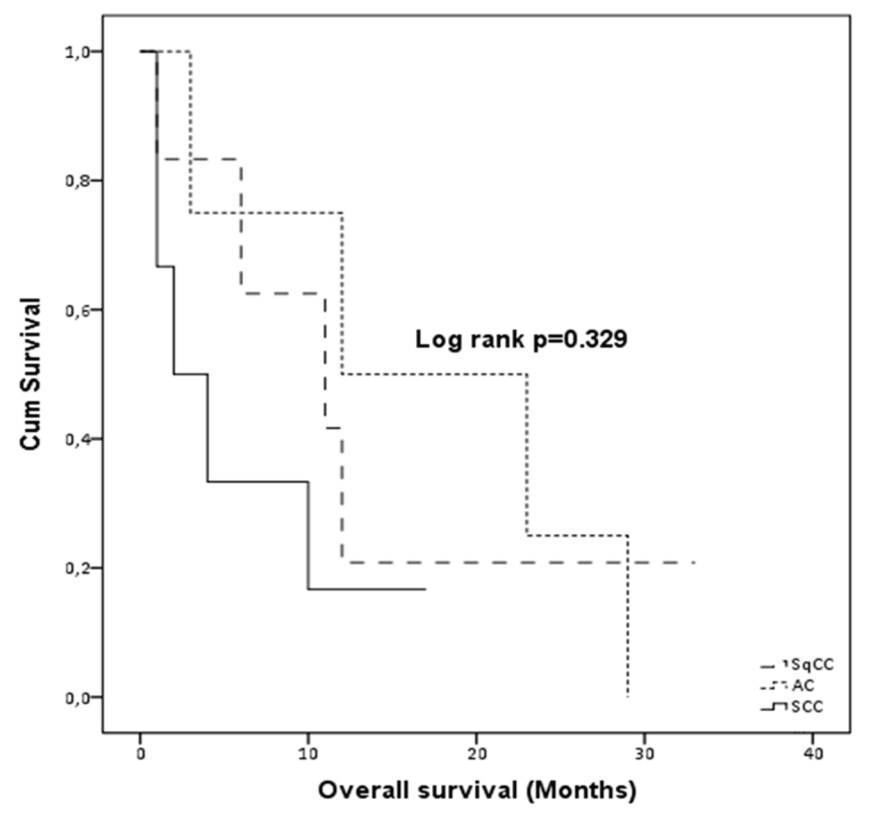

Figure 1. Survival according to histological groups AC: Adenocarcinoma, SCC: Small cell carcinoma, SqCC: Squamous cell carcinoma Table 2. The mean survival according to histological groups

\begin{tabular}{|l|l|l|l|l|}
\hline & $\begin{array}{l}\text { Median } \\
\text { (months) }\end{array}$ & SD & \multicolumn{2}{|l|}{$95 \% \mathrm{Cl}$} \\
\hline SqCC & 11.0 & 5.3 & 0.5 & 21.4 \\
\hline Adenocarcinoma & 12.0 & 10.0 & 0.2 & 31.6 \\
\hline SCC & 2.0 & 1.8 & 0.5 & 5.6 \\
\hline All patients & 10.0 & 4.6 & 0.8 & 19.1 \\
\hline
\end{tabular}

SCC: Small cell carcinoma, SqCC: Squamous cell carcinoma, SD: Standard deviation, $\mathrm{Cl}$ : Confidence interval cisplatin (15). In our study, there were two patients (50\%) with stage $4 \mathrm{AC}$ and two patients (50\%) underwent curative surgery. In our study, adjuvant therapy was administered to one patient (25\%) and palliative CT was administered to three patients (75\%). Platin combined with fluorouracil and gemcitabine were used as CT regimens. During the follow-up, all patients died due to cancer. In our study, the median OS in AC patients was 12 months. Bladder associated SCC is a rare, aggressive and poorly differentiated neuroendocrine neoplasm that represents less than $1 \%$ of all bladder cancers. There is no available randomized clinical study to guide the treatment of bladder SCC. Unlike UC, the presence or absence of muscle invasion in SCC has no effect on the treatment decisions because there is a high possibility of metastasis regardless of the tumor stage in SCC (16). In a retrospective study, 48 patients underwent surgery after neoadjuvant $\mathrm{CT}, 47$ patients underwent surgery, and then 21 of 47 patients were administered adjuvant CT. Mainly PE or ifosfamide + doxorubicin were used as neoadjuvant treatment regimen. The 5 -year survival was significantly longer in patients who were treated with neoadjuvant CT (79\% versus $20 \%)$. The benefit of adjuvant CT on survival could not be demonstrated (17). In a retrospective study with patients from Mayo Clinic between 1980 and 2005, the 5-year survival rate was found to be significantly higher in 18 patients who were given postoperative adjuvant CT compared to patients who were not given adjuvant CT ( $43 \%$ versus $20 \%$; $p=0.03$ ) (18).

Higher response rates were detected in patients with metastatic bladder SCC treated with $\mathrm{CT}$ regimen that were used in lung SCC (19). The median OS in bladder SCC was approximately 7-13 months $(19,20)$. Although systemic CT is the preferred treatment for metastatic disease, there is not enough data to guide the selection of the most appropriate regimen. In a prospective study, 12 patients with stage 4 disease (lymph node involvement was detected in only five patients) were treated with ifosfamide + doxorubicin, and PE, and complete response was obtained in three patients and surgical treatment could be performed. The median OS was found to be 13 months (19). In our presented study, there was one patient (16.7\%) with stage 2 SCC and 5 patients (83.3\%) with stage 4 SCC. One patient underwent curative surgery and received adjuvant PE. Two patients (33.3\%) were administered local RT at the time of diagnosis. The median OS in SCC was detected as 2 months in our study. The reasons for shorter survival in SCC patients in the present study could be due to the detection of ECOG performance score as 3 in 4 patients (66.7\%) and lack of CT in 2 patients due to lower ECOG performance. 


\section{CONCLUSION}

In conclusion, there is no available large prospective randomized clinical study to guide the treatment in non-urothelial bladder cancer. Based on these results, we suggest that patients diagnosed with NUBC should be included in prospective clinical studies.

\section{Ethics}

Ethics Committee Approval: This study was approved by the Ethics Committee of the İstanbul Okmeydanı Training and Research Hospital (approval no: 48670771-514.10).

Informed Consent: Patients were not required to give informed consent, because the study was retrospective and anonymous data were used, which were obtained after each patient agreed to treatment by written consent.

Peer-review: External and internal peer-reviewed.

\section{Authorship Contributions}

Surgical and Medical Practices: A.S., N.Y., Concept: A.S., O.C., Design: A.S., S.A., O.C., Data Collection or Processing: A.S., N.Y., C..G., Analysis or Interpretation: S.A., S.S., C.D., Literature Search: C.D., N.Y., C..G., Writing: A.S., S.A., S.S.

Conflict of Interest: No conflict of interest was declared by the authors.

Financial Disclosure: The authors declared that this study received no financial support.

\section{REFERENCES}

1. Dahm P, Gschwend JE. Malignant non-urothelial neoplasms of the urinary bladder: a review. Eur Urol 2003;44:672-81.

2. Ghoneim MA, Ashamallah AK, Awaad HK, Whitmore WF Jr. Randomized trial of cystectomy with or without preoperative radiotherapy for carcinoma of the bilharzial bladder. J Urol 1985;134:266-8.

3. Loh KP, Mondo E, Hansen EA, Sievert L, Fung C, Sahasrabudhe DM, et al. Targeted Therapy Based on Tumor Genomic Analyses in Metastatic Urachal Carcinoma. Clin Genitourin Cancer 2016;14:e449-52.

4. Cheng L, Pan CX, Yang XJ, Lopez-Beltran A, MacLennan GT, Lin H, et al. Small cell carcinoma of the urinary bladder: a clinicopathologic analysis of 64 patients. Cancer 2004;101:957-62.

5. Choong NW, Quevedo JF, Kaur JS. Small cell carcinoma of the urinary bladder. The Mayo Clinic experience. Cancer 2005;103:1172-8.
6. Ozbey I, Aksoy Y, Polat O, Biçgi O, Demirel A. Squamous metaplasia of the bladder: findings in 14 patients and review of the literature. Int Urol Nephrol 1999;31:457-61.

7. Kantor AF, Hartge P, Hoover RN, Fraumeni JF Jr. Epidemiological characteristics of squamous cell carcinoma and adenocarcinoma of the bladder. Cancer Res 1988;48:3853-5.

8. Scosyrev E, Yao J, Messing E.Urothelial carcinoma versus squamous cell carcinoma of bladder: is survival different with stage adjustment? Urology 2009;73:822-7.

9. James ND, Hussain SA, Hall E, Jenkins P, Tremlett J, Rawlings C, et al. Radiotherapy with or without chemotherapy in muscle-invasive bladder cancer. N Engl J Med 2012;366:1477-88.

10. Serretta V, Pomara G, Piazza F, Gange E. Pure squamous cell carcinoma of the bladder in western countries. Report on 19 consecutive cases. Eur Urol 2000;37:85-9.

11. Grignon DJ, Ro JY, Ayala AG, Johnson DE, Ordóñez NG. Primary adenocarcinoma of the urinary bladder. A clinicopathologic analysis of 72 cases. Cancer 1991;67:2165-72.

12. Xiaoxu L, Jianhong L, Jinfeng W, Klotz LH. Bladder adenocarcinoma: 31 reported cases. Can J Urol 2001;8:1380-3.

13. Zaghloul MS, Nouh A, Nazmy M, Ramzy S, Zaghloul AS, Sedira MA, et al. Long-term results of primary adenocarcinoma of the urinary bladder: a report on 192 patients. Urol Oncol 2006;24:13-20.

14. Galsky MD, lasonos A, Mironov S, Scattergood J, Donat SM, Bochner BH, et al. Prospective trial of ifosfamide, paclitaxel, and cisplatin in patients with advanced non-transitional cell carcinoma of the urothelial tract. Urology 2007;69:255-9.

15. Hong JY, Choi MK, Uhm JE, Park MJ, Lee J, Park SH, et al. Palliative chemotherapy for non-transitional cell carcinomas of the urothelial tract. Med Oncol 2009;26:186-92.

16. Patel SG, Stimson CJ, Zaid HB, Resnick MJ, Cookson MS, Barocas DA, et al Locoregional small cell carcinoma of the bladder: clinical characteristics and treatment patterns. J Urol 2014;191:329-34.

17. Lynch SP, Shen Y, Kamat A, Grossman HB, Shah JB, Millikan RE, et al. Neoadjuvant chemotherapy in small cell urothelial cancer improves pathologic downstaging and long-term outcomes: results from a retrospective study at the MD Anderson Cancer Center. Eur Urol 2013;64:307-13.

18. Kaushik D, Frank I, Boorjian SA, Cheville JC, Eisenberg MS, Thapa P, et al Long-term results of radical cystectomy and role of adjuvant chemotherapy for small cell carcinoma of the bladder. Int J Urol 2015;22:549-54.

19. Siefker-Radtke AO, Kamat AM, Grossman HB, Williams DL, Qiao W, Thall PF, et al. Phase II clinical trial of neoadjuvant alternating doublet chemotherapy with ifosfamide/doxorubicin and etoposide/cisplatin in small-cell urothelial cancer. J Clin Oncol 2009;27:2592-7.

20. Ismaili N, Heudel PE, Elkarak F, Kaikani W, Bajard A, Ismaili M, et al. Outcome of recurrent and metastatic small cell carcinoma of the bladder. BMC Urol 2009;9:4. 